\title{
La tutela dei diritti umani e le missioni di pace: Introduzione
}

\section{Federico Casolari, Luca Paladini}

Storicamente, 4 (2008).

ISSN: 1825-411X. Art. no. 20. DOI: 10.12977/stor551

Nel sistema della Carta delle Nazioni Unite (ONU), la tutela dei diritti fondamentali è chiamata ad assolvere una duplice funzione. Da un lato, essa costituisce per se un obiettivo primario dell'Organizzazione, riconosciuto in quanto tale nell'articolo 1 della Carta, e perseguito dagli organi dell'ente nell'esercizio delle rispettive funzioni. Dall'altro, essa rappresenta un'indispensabile condizione per il mantenimento della pace e della sicurezza internazionale, fine ultimo dell'Organizzazione.

Questo dualismo trova una felice sintesi in una formula che compare nel preambolo della Dichiarazione universale dei diritti umani, solennemente proclamata dall'Assemblea generale delle Nazioni Unite il 10 dicembre 1948, e - come noto - primo strumento a portata universale ad esser stato adottato per la tutela dei diritti individuali. II primo 'considerando' della Dichiarazione prevede, infatti, che «il riconoscimento della dignità inerente a tutti i membri della famiglia umana e dei loro diritti, uguali ed inalienabili, costituisce il fondamento della libertà, della giustizia e della pace nel mondo".

La previsione della Dichiarazione universale ha trovato molteplici riscontri nell'azione degli Stati membri dell'Organizzazione delle Nazioni Unite e dei suoi organi. Non vi è dubbio, però, che una delle manifestazioni della prassi più interessanti sia rappresentata dalle operazioni di mantenimento (o ristabilimento) della pace intraprese direttamente dall'ONU (i c.d. "caschi blu"), o svolte, previa autorizzazione ONU, da organizzazioni regionali (ad 
esempio dall'Organizzazione del Trattato dell'Atlantico del Nord, NATO, o dall'Unione europea), o, ancora, condotte al di fuori del quadro ONU ma in conformità con i suoi principi (è il caso di alcune operazioni dell'Unione europea che, pur non essendo previamente avallate dalle Nazioni Unite, debbono, per espressa previsione del Trattato sull'Unione europea, risultare conformi ai principi della Carta ONU).

In effetti, in quanto strumento per il mantenimento (o ristabilimento) della pace, tali operazioni concorrono direttamente a consolidare (o restaurare) uno standard accettabile di tutela dei diritti individuali nelle aree in cui sono chiamate ad agire. D'altra parte, le stesse operazioni, nella misura in cui si inseriscono nell'azione svolta dalle Nazioni Unite o dichiarano di ispirarsi ai suoi principi, dovrebbero ritenersi vincolate a garantire, nell'esercizio delle proprie funzioni, il rispetto dei diritti individuali e delle libertà fondamentali. Tale rispetto dovrebbe insomma rappresentare al contempo un fine ed un limite del loro operato.

Occorre tuttavia riconoscere che, soprattutto con riguardo al secondo aspetto evidenziato, si profilano questioni giuridiche che non hanno ancora trovato una definitiva sistemazione: si intende fare riferimento, per esempio, al tema del rapporto tra diritto internazionale umanitario e diritto internazionale dei diritti dell'uomo; al problema dell'applicazione extraterritoriale delle principali convenzioni internazionali sui diritti individuali; alla questione relativa all'attribuzione di una certa condotta allo Stato di nazionalità del singolo membro dell'operazione che ne è autore piuttosto che all'organizzazione internazionale nel cui ambito l'operazione è stata avviata.

Anche da questo punto di vista, il $60^{\circ}$ anniversario dell'adozione della Dichiarazione universale dei diritti dell'uomo rappresenta un'importante occasione per compiere una sistemazione generale della prassi relativa alle missioni di pace sin qui svolte e per tentare di fornire una valutazione d'insieme sulla posizione assunta da tali missioni rispetto al perseguimento della tutela dei diritti fondamentali. 